\title{
REVIEW
}

\section{Clinical review: Ethics and end-of-life care for critically ill patients in China}

\author{
Li Bin Li
}

\begin{abstract}
Critical care medicine in China has made great advances in recent decades. This has led to an unavoidable issue: end-of-life ethics. With advances in medical technology and therapeutics allowing the seemingly limitless maintenance of life, the exact time of death of an individual patient is often determined by the decision to limit life support. How to care for patients at the end of life is not only a medical problem but also a social, ethical, and legal issue. A lot of factors, besides culture, come into play in determining a person's ethical attitudes or behaviors, such as experience, education, religion, individual attributes, and economic considerations. Chinese doctors face ethical problems similar to those of their Western counterparts; however, since Chinese society is different from that of Western countries in cultural traditions, customs, religious beliefs, and ethnic backgrounds, there is a great difference between China and the Western world in regard to ethics at the end of life, and there is also a huge controversy within China.
\end{abstract}

\section{Introduction}

China has made great socioeconomic advances in the past 30 years, and more and more hospitals have established a modern ICU, resulting in an obvious reduction of mortality of critically ill patients $[1,2]$. Critical care is an integral part of hospital care, and the ICU is a setting where patients are given the most technologically advanced life-sustaining treatments. These treatments are expensive and resource-intensive but can sustain life even when there is no cure or hope. The ICU is, however, also a setting where death is common and end-oflife care is frequently provided $[2,3]$. Since the focus in ICUs is on sustaining life, the delivery of high-quality

Correspondence: lilibinhz@163.com

Department of Critical Care Medicine, Second Affiliated Hospital of Zhejiang University College of Medicine, 88 Jiefang Road, Hangzhou 310009, China end-of-life care can be particularly challenging, and clinicians often find the dual responsibilities of saving lives and delivering end-of-life care difficult. Given the nature of critical care, difficult decisions often need to be made about the usefulness of life-supporting treatments, in terms of not only the probability of survival but also the quality of life associated with survival [3].

It is an inescapable fact that limiting life support is sometimes necessary and acceptable in patients who are hopelessly critically ill [4-6]. Indeed, with advances in medical technology allowing the seemingly limitless maintenance of life, the exact time of death of an individual patient is often determined by the decision to limit life support. However, end-of-life decisions are not easy and can be influenced by various factors, including the age and experience of the doctor, the country and culture of origin, the attitudes of the country/area where the doctor is currently working, and religious beliefs [7]. Chinese ICU doctors face ethical problems similar to those of their European and American counterparts, but there are also differences [3-6]. This article will discuss general aspects surrounding these ethics and the end-oflife care for critically ill patients in China, and, where possible, special reference will be given to known international/cultural differences. In a sense, an advance in modern medicine is indeed a double-edged sword, and some medical technology breakthroughs are often the source of many of the challenges we face. This is particularly prominent in the ICU in China.

\section{Ethics of the end of life in China: status quo and dilemma}

Owing to modern medicine, life-support technology helps to prolong the end of life of critically ill patients in the biological sense, but still death cannot be avoided ultimately. A large proportion of deaths in the US occur in the ICU or after a stay in the ICU [8], and approximately one in five Americans dies in an ICU [9]. The ratio is estimated to be increasing year by year. Despite the lack of 
precise statistics, the number of patients who die in the ICU in China is rising every year $[2,6,10]$.

The aging population in many countries will increase the importance of these end-of-life issues in the future. As China has made great social and economic advances in past decades and has entered the aging society, the aging population (> 60 years old) accounting for 14.3\% of the total population, population aging is also becoming an increasingly severe problem. At present, China's aging population has increased to approximately 200 million and is larger than that of the whole of Europe $[11,12]$. So the number of older patients is increasing with time. Meanwhile, thousands of hospitals have established an ICU in the last 20 years, and this has brought about a grim problem $[4,10]$. For example, more and more older patients, who would have died within a short period of time in the past, may now achieve longterm survival $[4,5]$. For these patients, intensive care just means extended life or postponed death. These hopeless patients occupy an already scarce medical resource, and this makes it impossible for some critically ill patients to receive effective treatment [6]. Owing to the influence of Chinese traditional ethics and the lack of corresponding laws and regulations, overtreatment of hopeless patients frequently takes place. In fact, it is a futile treatment $[4,10]$. Chinese society needs to develop approaches to address the appropriate delivery of critical care to the increasing population of older people, especially those with chronic life-limiting disease $[4,5,13]$.

With the advancement of science and technology for life support, demands for intensive care services have been escalating. However, owing to limited resources, difficult end-of-life ethical issues often arise in the ICU. China is facing the same problem [13,14]. According to the existing Chinese guidelines for ICU admission, ICUs should select patients who are likely to benefit from intensive care [15]. But the reality is that it is very difficult to implement these guidelines strictly. For example, China is a human-relations society, and sometimes doctors find it difficult to deny patients who have no indications for ICU admission [4,13]. They are worried that their denial would deteriorate the relationship between doctors and patients [16].

In China, only the patients' family members or surrogates can make the decision to limit treatment. At present, only a small number of families informed of treatment for their loved ones in hopeless cases take the initiative to make the decision to withdraw treatment, in order to alleviate the suffering of patients [5]. Most families that make the decision to withdraw treatment do so for economic reasons, and most of them come from rural areas $[5,6]$. They have to pay a higher proportion of medical costs by themselves, and these costs can leave them in poverty $[6,14,17]$. Xue and Zhu [6] showed that economic condition was the key factor in deciding whether to forgo treatment.

Because more and more doctor-patient disputes have appeared in recent years, Chinese doctors tend to protect themselves by being passive in making treatment decisions $[17,18]$. It is very important to establish the standards concerning forgoing life-sustaining treatments in critically ill patients. Sometimes giving up treatment is also a relief for patients and their families. Although some Chinese doctors are aware that giving up useless treatment is a wise choice, the possibility of involvement in medical disputes and undertaking legal liability means that they seldom advise families to withdraw treatment [4-6,18]. Owing to a lack of communication, families often are unable to be well informed of the severity of the disease or to get reasonable advice in making treatment decisions $[6,19]$. A recent multicenter study about the attitudes of Chinese ICU doctors toward ethical problems showed that there were a number of differences between Chinese and European ICU doctors in their ethical attitudes and relevant behaviors. For example, the use of do not resuscitate (DNR) orders was low: the rate of Chinese doctors not applying DNR orders at all was $53 \%$ [20] compared with less than $5 \%$ of doctors in Northern and Central Europe, less than 10\% in Southern Europe [21], and only 5\% in Hong Kong [22]. Interestingly, the reported use of limitation of lifesustaining therapy, both withholding (54\%) and withdrawing (32\%), by Chinese ICU doctors was also low and was substantially lower than that reported in Europe in 1990 (withholding 83\% and withdrawing 63\%) and 1999 (withholding 93\% and withdrawing 77\%) [23,24]. Although a likely explanation for these differences is cultural heritage, other potential explanations exist [20].

\section{Influence of traditional Chinese culture on end-of-life decision-making}

There are some common issues between Chinese and Western bioethics, such as euthanasia, physician-assisted suicide, brain death, organ transplantation, artificial insemination and in vitro fertilization, and health-care resource distribution. However, some problems are unique to China [25,26]. Attitudes and behaviors to the end of life are heavily dependent on cultural influences [27]. The various religions' views on end-of-life decisions are not identical. Most Chinese people have no religious beliefs, and Chinese doctors are no exception $[20,28]$. According to a survey, only about $10 \%$ of Chinese people are affiliated with religions, which include Buddhism, Christianity, and Islam [28]. However, religious beliefs play an important part when a person determines whether to limit life support for the critically ill patient. Confucianism is not considered a religion by most Chinese people. The moral perspective is influenced primarily 
by Confucianism but also by Taoism and Buddhism [29]. With this mixture of different religions and philosophies in one population, very diverse opinions and dilemmas can be encountered [27]. For example, Confucianism says 'no' to withholding and withdrawing of lifesustaining therapy for patients at the end of life. However, Buddhism and Taoism say 'yes', and Catholics, Protestants, and Muslims have similar viewpoints to Buddhism and Taoism [27].

In China, patients often lack autonomy and therefore their wishes sometimes do not get the corresponding respect [30]. When a patient enters the ICU, he or she often has no chance to make a will. Ordinary Chinese people are reluctant to make a will because they think it is inauspicious. Once patients are critically ill and incurable, their families do not know the true wishes of their loved ones, and this influences medical decision-making $[4,5,14]$. Sometimes a few surrogates verbally agree to forgo treatment but in the end refuse to forgo treatment in writing because of the emotional, ethical, and social implications as well as public opinion [4-6]. Social reaction can be severe if a terminally ill older patient is allowed to die or is denied life-saving intervention. The offspring might be blamed by third parties for violating the principle of filial piety, the doctors might be accused of malpractice or murder, and supporters might be criticized for being anti-humanitarian or violating the principle of saving the dying $[5,14]$. It has been suggested that the Confucian value of filial piety makes it difficult for adult children to forgo life-prolonging treatment for a parent. Filial piety encourages offspring to 'rescue with all their strength' and to save a parent regardless of cost [31]. It has also been suggested that the importance of rescuing at any cost potentially creates conflict for the physician, who may believe that the limitation of treatment is in the patient's interests but fears that discussion with the family would create circumstances for disharmony, and harmony is a value that doctors traditionally are expected to uphold [31,32].

Chinese bioethics is a family-based and harmonyoriented ethical system embedded in the Confucian way of life. It has characterized Chinese health care and medical practice for thousands of years. Evidently, there are striking differences that distinguish this bioethics from the individual-based and autonomy-oriented bioethics that emerged in the West $[25,26,32]$.

In Chinese culture, familial relationships are stressed more than an individual's rights. A family will assume responsibility, including medical decision-making, for an individual's care $[20,30,32]$. This extends to the amount of information given to a patient as it is thought necessary to protect and not overburden him or her. Conveying bad news to a patient without the family's consent may result in conflict, and much discussion may be involved in clarifying issues so that a compromise can be reached. As a consequence, a patient may not be in full knowledge of his or her medical condition. The perceived need to maintain harmony and avoid conflict in the doctor-patient relationship during the stressful period at the end of life may also be an important contributing factor [17,19,32]. However, in China, the Ministry of Health, which is responsible for running all hospitals, has not published guidelines concerning the limiting of life support in critically ill patients. Nowadays, hospital ethics committees have been set up in all of the large-scale hospitals in China and are responsible for ethics education and ethics review consultation. But they do not participate in ethics decisions for patients at the end of life $[14,26,33]$. The Chinese Society of Critical Care Medicine and the Chinese Society of Medical Ethics have not yet issued relevant guidelines [14,26].

In Chinese culture as a whole, the topic of death is generally taboo, and this prohibits the physicians from discussing death in much detail with the patient or the patient's family $[17,26,32]$. The maintenance of hope is considered very important in the care of the dying, as hope prevents suffering by avoiding despair. For example, in China, there has been great controversy on brain death. Brain death legislation was met with a great deal of resistance $[14,17,34]$. Brain death has been extensively discussed in medical and ethical circles, and diagnostic criteria have been issued by the Ministry of Health. Most Chinese doctors, however, do not believe that brain death will be approved by the government or legislative authorities in a short time.

Organ transplantation in China has expanded rapidly in the past 20 years. According to official statistics, more than 1.5 million people in China need a transplant every year, but only 10,000 are able to undergo surgery. More than $99 \%$ of patients need to wait for organ donation and most of them will die while waiting [35]. However, the rapid expansion of the capacity to do transplantations has not been accompanied by the development of an ethical system for recovering organs from those who die in hospitals while on life support, as is international practice. The use of executed prisoners as the main source of organs is a morally reprehensible practice, for which the Chinese government and medical circles have been strongly criticized [36]. At present, with the refinements to the legal system and the willingness to improve its international reputation, the Chinese government is trying to discourage the practice $[23,36]$. Because of the view of traditional Chinese culture on the preservation of an intact body, most Chinese people have a negative attitude toward organ donation, and this is an important reason why transplant activities have been limited in China. Organ transplantation is not just a simple medical technology problem but rather a complex process involving medical and social ethics in practice $[37,38]$. 
Euthanasia, which is still illegal in China, is a topic that is hardly discussed in medical and legal circles. Even though patients and their families convey their strong desire for euthanasia, it is still prohibited. In China's judicial system, almost all cases of euthanasia have resulted in a guilty verdict [34]. The difficulty of practicing euthanasia on the older population originates from factors in the traditional ethics that exert a far-reaching influence on Chinese social philosophy: ancestor worship, the patriarchal clan system, and the Confucian principle of filial piety. In traditional thought, according to the principle of filial piety, children should extend the lives of their parents as long as possible. Because the corpse and the organs are considered sacred, it is very difficult to obtain cadavers for experimentation or organs for transplantation [37-39]. Although euthanasia has not been widely accepted in China, more and more people have realized that euthanasia is a relief for some critically ill patients, and many prominent figures have begun to call for the legalization of euthanasia [40].

\section{Characteristics of Chinese bioethics and the differences between Chinese and Western bioethics}

Bioethics does not formally exist within traditional Chinese culture but was borrowed and introduced from Western countries [39]. Chinese bioethics share some similarities with Western bioethics, but the former has some different topics, such as health inequality in health-care reform and the doctor-patient relationship, and a different model of informed consent [22,30,41]. Therefore, Chinese bioethics is different from that of the Western world in its philosophical basis and culture context, which is influenced by Confucianism, Taoism, and Buddhism [25,27,32].

Bioethics in China does have some different characteristics $[41,42]$. The first one is beneficence, which is still commonly accepted as the number one principle when dealing with clinical issues. Patient-centered beneficence is the most important context in truth-telling, where the good and benefits of the patients are interpreted and represented by close family members under the guidance and suggestion of doctors [42,43]. As a result, the decision to communicate the truth about a diagnosis or prognosis to the patient depends on considerations of the patient's condition, the likely impact on the patient, and the family's wishes in the matter [42]. In a word, the general justification for deception must be the patient's best interests. One study showed that the majority of Chinese ICU doctors indicated that they would give their patients specifically tailored information rather than complete medical information [20]. Especially, patients in the ICU, who have been intubated or are already in a coma, tend to lose the opportunity to make a will [5].

The second characteristic of Chinese bioethics is family-oriented autonomy and the family model of informed consent. In China, informed consent in medical practice is not given by the individual patient but by the family [42-44]. Doctors frequently inform the families rather than the patient in medical decision-making. This stands in contrast with the Western model, in which individual autonomy of the patient is more highly respected. This Chinese paradigm of informed consent can be called a doctor-family-patient relationship, instead of doctor-patient relationship in Western countries [44]. A special family autonomy in China favors collective decision-making over respecting the will of individual patients and regards paternalism as being supported by Confucian culture. Consequently, a general difference between Western and Chinese doctors is very clear: Western doctors would need a special reason to justify not telling the truth to the patient, whereas Chinese doctors would need a special reason to justify telling the truth to the patient $[44,45]$. This approach may be explained by Confucianism and later philosophical interpretations of the doctor-patient relationship. These emphasize the need to avoid transmitting unnecessary damaging information to the patient, but only when believed to be in the best interests of the patient, with the aim of preventing disharmony-an important goal given the traditional Chinese value of creating and maintaining harmonious human relationships [25,46].

In a word, Confucianism is one of the important points to comprehend the traditional Chinese doctor's behavior and medical practice, and it also sheds light on modern Chinese medical practice. To a large extent, Chinese bioethics is profoundly influenced by Confucianism. The main theme of Confucianism is ren (humanness), a theory of virtue ethics and deontology. Doctors are responsible to do good for patients.

\section{Conclusions}

China is undergoing a significant shift from traditional ways to modernization in economic, social, and political fields. Chinese ICU doctors face ethical problems similar to those of their European and American counterparts, but there are also differences. Whether Chinese ethics can be meaningfully blended with Western ethics is a question that medical ethicists cannot afford to ignore in the interests of the cross-cultural assimilation of medical practice. After all, China is a country with thousands of years of cultural tradition. We should be aware of its special situation. Ethical and behavioral differences between cultures serves to remind us to strive to understand the foundations of these differences and develop strategies to deal with them. Owing to China's current 
economic development level and social medical insurance system, it is beneficial to deliberate upon ethical issues in the treatment of the end-of-life patients, for more rational allocation of limited medical resources.

In the future, it will be very important and necessary to do more and better research to support the development of Chinese bioethics. Chinese doctors need corresponding guidelines, and even legal assistance, to solve some ethical issues in practice.

\section{Abbreviations}

DNR: Do not resuscitate.

\section{Competing interests}

The author declares that he has no competing interests.

\section{Published: 04 Dec 2013}

\section{References}

1. Du B, Xi XM, Chen DC, Peng JM: Critical care medicine in mainland China. Crit Care 2010, 14:206-211.

2. Wang Y, Ma PL: The emergence and development of ICU. Med Philos 2006, 27:7-9.

3. Curtis JR, Vincent JL: Ethics and end-of-life care for adults in the intensive care unit. Lancet 2010, 16:347-353.

4. Wang XS, Yang XL, Liu W: Difficulties of ICU in the elderly society. Med Philos 2009, 30:62-64.

5. Wang XS, Yang XL, Liu W: Withdrawal of life-sustaining treatments in the ICU. Med Philos 2009, 30:66-68.

6. Xue XY, Zhu JH: Analysis of ICU patients' outcome in different end-of-life care and its influence factors. Med Philos 2011, 32:27-28.

7. Vincent IL: Cultural differences in end-of-life care. Crit Care Med 2001, 2: N52-N55.

8. The SUPPORT Principal Investigators: A controlled trial to improve care for seriously ill hospitalized patients: the study to understand prognoses and preferences for outcomes and risks of treatments (SUPPORT). JAMA 1995, 274:1591-1598.

9. Angus DC, Barnato AE, Linde-Zwirble WT, Weissfeld LA, Watson RS, Rickert T, Rubenfeld GD, Robert Wood Johnson Foundation: Use of intensive care at the end of life in the United States: an epidemiologic study. Crit Care Med 2004, 32:638-643.

10. Wang XY, Liu YP: Epidemiological survey of ICU patients died. Chin Postgrad Med 2007, 30:37-39.

11. National Bureau of Statistics of China: Statistical communique of the People's Republic of China on the 2012 National Economic and Social Development. [http://www.stats.gov.cn/tjgb/ndtjgb/qgndtjgb/t20130221_402874525.htm].

12. China's aging population to double by 2053. [http://www.chinadaily.com.cn/ china/2012-10/23/content_15837794.htm].

13. Wang YS, Liu DX: On standard of ICU at present in China. Med Philos 2006 27:26-27.

14. Liu YP, Wan XY: Ethic issues about the decision making of the patients at the end of life in the intensive care unit. Med Philos 2011, 32:4-6.

15. Society of Critical Care Medicine of Chinese Medical Association: ICU construction and management guide (2006). Chin Crit Care Med 2006, 7:387-388.

16. China to enhance hospital security amid rising doctor-patient tensions. [http:// news.xinhuanet.com/english/china/2012-02/24/c 131430425.htm].

17. Guan XX, Yang GB, Chen LB: Ethical reflection on the treatment of terminal stage patients. Chin Med Ethics 2010, 23:26-27.

18. Wang XY, Liang LZ, Lu Y, Wu LN: Research on factors and counter measure of doctor-patient relationship in view of hospital and staff. Chin Med Ethics 2008, 21:33-36.

19. Liu YP, Wan XY: Communication between physicians and patients play an important role on improving end-of-life care in ICU. Med Philos 2007 28:29-30.

20. Weng L, Joynt GM, Lee A, Du B, Leung P, Peng J, Gomersall CD, Hu X, Yap HY, Chinese Critical Care Ethics Group: Attitudes towards ethical problems in critical care medicine: the Chinese perspective. Intensive Care Med 2011, 37:655-664.

21. Azoulay E, Metnitz B, Sprung CL, Timsit JF, Lemaire F, Bauer P, Schlemmer B, Moreno R, Metnitz P, SAPS 3 investigators: End-of-life practices in 282 intensive care units: data from the SAPS 3 database. Intensive Care Med 2009, 35:623-630.

22. Yap HY, Joynt GM, Gomersall CD: Ethical attitudes of ICU physicians in Hong Kong. Hong Kong Med J 2004, 10:244-250.

23. Vincent $\mathrm{J}$ : European attitudes towards ethical problems in intensive care medicine: results of an ethical questionnaire. Intensive Care Med 1990, 16:256-264.

24. Vincent JL: Forgoing life support in western European intensive care units: the results of an ethical questionnaire. Crit Care Med 1999. 27:1626-1633

25. Mao XZ: Considerations on research and development in bioethics in modern China. Chin Med Ethics 2006, 19:22-24.

26. Wan X, Guo Y. The current difficulties and development trends of Chinese bioethics. Chin Med Ethics 2012, 25:563-566.

27. Bülow HH, Sprung CL, Reinhart K, Prayag S, Du B, Armaganidis A, Abroug F, Levy MM: The world's major religions' points of view on end-of-life decisions in the intensive care unit. Intensive Care Med 2008, 34:423-430

28. The State Council Information Office of the People's Republic of China: Situation of religious freedom in China. [http://www.china.com.cn/ch-book/ zongjiao/zongjiao.htm]

29. Bowman KW, Hui EC: Bioethics for clinicians: Chinese bioethics. Can Med Assoc J 2000, 163:1481-1485.

30. Chen XY, Fan RP: The family and harmonious medical decision making: cherishing an appropriate Confucian moral balance. J Med Philos 2010, 35:573-586.

31. Blumenthal D, Hsiao W: Privatization and its discontents - the evolving Chinese health care system. N Engl J Med 2005, 353:1165-1170.

32. Cong Y: Ethical challenges in critical care medicine: a Chinese perspective. J Med Philos 1998, 23:581-600.

33. Li EC: Bioethics in China. Bioethics 2008, 22:448-454.

34. Jia GY: Traditional thanatopsis towards euthanasia and hospice. Chin Med Ethics 2011, 24:224-225.

35. China expands human organ donation pilot. [http://news.xinhuanet.com/ english/indepth/2013-01/27/c_132131226.htm].

36. China to end reliance on organ donations from condemned prisoners. [http:// news.xinhuanet.com/english/china/2012-11/23/c_131994014.htm].

37. WU AMS, Lu LS: Cognitive obstacles against organ donation: the influence of negative attitudes, norms, and traditional beliefs on Chinese people's intention to donate organs after death. J Community App/ Soc Psychol 2011, 21:87-93.

38. Lu J, Ye ZQ, Li Q: Ethical issues and countermeasure on organ transplant. Chin Med Ethics 2006, 19:37-39.

39. Zhang YM, Meng XJ: Ethical dilemmas in the domestic shortage of cadaveric donors from the viewpoint of value. Chin Med Ethics 2012 25:567-568

40. Wang QY: Advocates call for euthanasia law. [http://www.chinadaily.com.cn/ china/2012-12/04/content_15982493.htm].

41. Gao W, Zhang JP: Cultural reflection on the localization of bioethics in China. Chin Med Ethics 2009, 22:47-49.

42. Fan RP, Li BF: Truth telling in medicine: the Confucian view. J Med Philos 2004, 29:179-193.

43. Dong PP, Wang LY: The influence of Chinese family culture on the application of informed consent. Chin Med Ethics 2011, 24:262-264.

44. Cong Y: Doctor-family-patient relationship: the Chinese paradigm of informed consent. J Med Philos 2004, 29:149-178.

45. Fan RP: Self-determination vs. family-determination: two incommensurable principles of autonomy: a report from East Asia. Bioethics 1997, 11:309-322.

46. Yan JJ, Shen LH, Zeng YP: Ethical investigation of the main body in the implementation of informed consent in hospitals. Chin Med Ethics 2011, 24:743-746

\section{$10.1186 / \mathrm{cc} 13140$}

Cite this article as: Li: Clinical review: Ethics and end-of-life care for critically ill patients in China. Critical Care 2013, 17:244 